\title{
Leverrier-Takeno Coefficients for the Characteristic Polynomial of a Matrix
}

\author{
I.Guerrero-Moreno $^{1}$, J. López-Bonilla ${ }^{1}$, J. Rivera-Rebolledo ${ }^{2}$ \\ ${ }^{1}$ ESIME-Zacatenco, Instituto Politécnico Nacional (IPN) \\ Anexo Edif.3,Col. Lindavista 07738, México DF, E-mail: jlopezb@ipn.mx \\ ${ }^{2}$ Depto. de Física, Escuela Superior de Física y Matemáticas, IPN \\ Edif. 9, Zacatenco, Col. Lindavista 07738, México DF
}

Abstract: It is shown for any $\underset{\sim}{A} n$ an elementary method, in terms of the spur of $\underset{\sim}{A}{ }^{k}, k=1, \ldots, n$, to determine the coefficients of its characteristic polynomial.

\section{Introduction}

For arbitrary $\underset{\sim}{A}$ non , a matrix $(\underset{\sim}{A-\lambda I})$ can be constructed with a Characteristic Polynomial [1-3] given by:

$$
(-1)^{n} \operatorname{det}(\underset{\sim}{A-\lambda I})=\lambda^{n}+a_{1} \lambda^{n-1}+a_{2} \lambda^{n-2}+\ldots++a_{n-1} \lambda+a_{n}=0
$$

whose roots are the eigenvalues of $\underset{\sim}{\sim}$. There are mentioned in [4] the different techniques to get explicitely the coefficients $a_{j}, j=1, \ldots, n$, but here it is only considered the famous method of Leverrier [5] - Takeno [6] which determines $a_{j}$ via the expressions [7-12]:

$$
\begin{gathered}
a_{1}=-s_{1} \quad, 2 ! a_{2}=\left(s_{1}\right)^{2}-s_{2}, 3 ! a_{3}=-\left(s_{1}\right)^{3}+3 s_{1} s_{2}-2 s_{3}, \\
4 ! a_{4}=\left(s_{1}\right)^{4}-6\left(s_{1}\right)^{2} s_{2}+8 s_{1} s_{2}+3\left(s_{2}\right)^{2}-6 s_{4}, \\
5 ! a_{5}=-\left(s_{1}\right)^{5}+10\left(s_{1}\right)^{3} s_{2}-20\left(s_{1}\right)^{2} s_{3}+15 s_{1}\left[2 s_{4}-\left(s_{2}\right)^{2}\right]+20 s_{2} s_{3}-24 s_{5},
\end{gathered}
$$

where $s_{j}$ represents the spur of the $j$-th power of $\mathcal{A}$ :

$$
s_{j}=\operatorname{tr} \stackrel{\sim}{A}^{j} \quad, \quad j=1,2, \ldots, n
$$

that is, $s_{1}=\operatorname{tr} \underset{\sim}{A}, s_{2}=\operatorname{tr} \underset{\sim}{A^{2}}$, etc. The formulae (2) result when employing $a_{1}=-s_{1}$ in the recurrence relation:

$$
r a_{r}+s_{1} a_{r-1}+s_{2} a_{r-2}+\ldots+s_{r-1} a_{1}+s_{r}=0 \quad, \quad r=1, \ldots, n .
$$


Furthermore, if we let $\lambda=0$ in (1), it is obtained:

$$
a_{n}=(-1)^{n} \operatorname{det} \underset{\sim}{A} .
$$

Here it is shown an elementary development, alternative to (4), in order to construct expressions (2), which may be interesting when teaching Linear Algebra.

\section{Leverrier-Takeno Coefficients}

To reproduce (2), the basic idea consists in generating $a_{j}$ by means of products $s_{i_{1}} s_{i_{2}} \ldots s_{i_{k}}$ such that $\sum_{c=1}^{k} i_{c}=j$, which will be illustrated for $n=2,3$ y 4 , from where it will become evident how the method is applied for arbitrary $n$.

Let $n=2$, then $a_{2}$ will have the structure:

$$
a_{2}=c_{1} s_{1} s_{1}+c_{2} s_{2}=c_{1}\left(s_{1}\right)^{2}+c_{2} s_{2},
$$

where the quantities $c_{1}$ and $c_{2}$ are determined by selecting particular forms of $\mathcal{A}$, in fact:

If $\underset{\sim}{A}=\left(\begin{array}{ll}1 & 0 \\ 0 & 0\end{array}\right)$, then $\underset{\sim}{A^{2}}=\underset{\sim}{A} \quad \therefore \quad s_{1}=s_{2}=1, a_{2} \stackrel{(5)}{=} \operatorname{det} \underset{\sim}{A}=0$, so (6) implies:

$$
c_{1}+c_{2}=0
$$

and for $\underset{\sim}{A}=\left(\begin{array}{ll}1 & 0 \\ 0 & 1\end{array}\right)$ we have $\underset{\sim}{A^{2}}=\underset{\sim}{A}=\underset{\sim}{I} \quad \therefore \quad s_{1}=s_{2}=2, a_{2}=1$, and because (6) it results:

$$
2 c_{1}+c_{2}=\frac{1}{2}
$$

which together with (7.a) gives $c_{1}=-c_{2}=\frac{1}{2}$, then (6) reproduces the expression for $a_{2}$ shown in (2).

If $n=3$, the coefficient $a_{3}$ will take the form:

$$
a_{3}=c_{1} s_{1} s_{1} s_{1}+c_{2} s_{1} s_{2}+c_{3} s_{3}=c_{1}\left(s_{1}\right)^{3}+c_{2} s_{1} s_{2}+c_{3} s_{3},
$$

which can be applied to the following matrices:

$$
\underset{\sim}{A}=\left(\begin{array}{lll}
1 & 0 & 0 \\
0 & 0 & 0 \\
0 & 0 & 0
\end{array}\right) \quad, \quad \underset{\sim}{A^{3}}=\underset{\sim}{A^{2}=\underset{\sim}{A}} \quad \therefore \quad s_{1}=s_{2}=s_{3}=1, a_{3}=-\operatorname{det} \underset{\sim}{A}=0,
$$

and (8) leads to:

$$
c_{1}+c_{2}+c_{3}=0
$$

Similarly, 


$$
\begin{aligned}
& \underset{\sim}{\sim}=\left(\begin{array}{lll}
1 & 0 & 0 \\
0 & 1 & 0 \\
0 & 0 & 0
\end{array}\right) \quad: \quad 4 c_{1}+2 c_{2}+c_{3}=0, \\
& \underset{\sim}{A}=\left(\begin{array}{lll}
1 & 0 & 0 \\
0 & 1 & 0 \\
0 & 0 & 1
\end{array}\right) \quad: \quad 9 c_{1}+3 c_{2}+c_{3}=-\frac{1}{3},
\end{aligned}
$$

and the solution to the system (9.a,b,c) is $c_{1}=-\frac{1}{6}, c_{2}=\frac{1}{2}, c_{3}=-\frac{1}{3}$, which in (8) implies the corresponding expression (2).

For $n=4$ :

$$
\begin{aligned}
a_{4}= & c_{1} s_{1} s_{1} s_{1} s_{1}+c_{2} s_{1} s_{3}+c_{3} s_{2} s_{2}+c_{4} s_{1} s_{1} s_{2}+c_{5} s_{4}, \\
& =c_{1}\left(s_{1}\right)^{4}+c_{2} s_{1} s_{3}+c_{3}\left(s_{2}\right)^{2}+c_{4}\left(s_{1}\right)^{2} s_{2}+c_{5} s_{4},
\end{aligned}
$$

therefore:

$$
\begin{aligned}
& \underset{\sim}{\sim}=\left(\begin{array}{llll}
1 & 0 & 0 & 0 \\
0 & 0 & 0 & 0 \\
0 & 0 & 0 & 0 \\
0 & 0 & 0 & 0
\end{array}\right) \quad: \quad c_{1}+c_{2}+c_{3}+c_{4}+c_{5}=0 \\
& \underset{\sim}{\sim}=\left(\begin{array}{llll}
1 & 0 & 0 & 0 \\
0 & 1 & 0 & 0 \\
0 & 0 & 0 & 0 \\
0 & 0 & 0 & 0
\end{array}\right) \quad: \quad 8 c_{1}+2\left(c_{1}+c_{3}\right)+4 c_{4}+c_{5}=0 \\
& \underset{\sim}{\sim}=\left(\begin{array}{cccc}
1 & 0 & 0 & 0 \\
0 & -1 & 0 & 0 \\
0 & 0 & 0 & 0 \\
0 & 0 & 0 & 0
\end{array}\right): \quad 2 c_{3}+c_{5}=0 \\
& \underset{\sim}{\sim}=\left(\begin{array}{llll}
1 & 0 & 0 & 0 \\
0 & 1 & 0 & 0 \\
0 & 0 & 1 & 0 \\
0 & 0 & 0 & 0
\end{array}\right): \quad 27 c_{1}+3\left(c_{2}+c_{3}\right)+9 c_{4}+c_{5}=0, \\
& \underset{\sim}{A}={\underset{\sim}{I}}_{4 \times 4} \quad: \quad 64 c_{1}+4\left(c_{2}+c_{3}\right)+16 c_{4}+c_{5}=\frac{1}{4},
\end{aligned}
$$

the system (11) admits the solution $c_{1}=\frac{1}{24}, c_{2}=\frac{1}{3}, c_{3}=\frac{1}{8}, c_{4}=c_{5}=-\frac{1}{4}$, then (10) gives the formula for $a_{4}$ of (2). 
From the previous examples it is clear the way to apply the method for any $n$, and in this manner to reproduce the Leverrier-Takeno relations for the coefficients of the Characteristic Polynomial of an arbitrary matrix.

\section{REFERENCES}

1. D.T. Finkbeiner, Introduction to matrices and linear transformations, W.H. Freeman, San Francisco USA (1966)

2. C. Lanczos, Applied analysis, Dover, New York (1988)

3. R.A. Horn and Ch. R. Johnson, Matrix analysis, Cambridge University Press (1990)

4. A.S. Householder and F.L. Bauer, Numerische Math. 1 (1959) 29-37

5. U. J. J. Leverrier, J. de Math. Pures Appl. Série 1, $\underline{5}$ (1840) 220-254

6. H. Takeno, Tensor N.S. $\underline{3}$ (1954) 119-122

7. A.N. Krylov, Bull de l’Acad. Sci. URSS 7 , No.4 (1931) 491-539

8. P. Horst, Ann. Math. Stat. $\underline{6}$ (1935) 83-84

9. H. Wayland, Quart. Appl. Math. 2 (1945) 277-306

10. E.B. Wilson, J.C. Decius and P.C. Cross, Molecular vibrations, Dover, New York (1980) 216-217

11. J. López-Bonilla, J. Morales, G. Ovando and E. Ramírez G., Proc. Pakistan Acad. Sci. $\underline{43}$, No.1 (2006) 47-50

12. J. Caltenco, J. López-Bonilla and R. Peña R., Educatia Matematica $\underline{3}$, No.1-2 (2007) 107-112 